\title{
El alga marina Sargassum (Sargassaceae): una alternativa tropical para la alimentación de ganado caprino
}

\author{
M. Casas-Valdez ${ }^{1 *}$, H. Hernández-Contreras ${ }^{2}$, A. Marín-Álvarez ${ }^{1}$, R.N. Aguila-Ramírez ${ }^{*}$, \\ C.J. Hernández-Guerrero ${ }^{1 *}$, I. Sánchez-Rodríguez ${ }^{1 *} \&$ S. Carrillo-Domínguez ${ }^{3}$ \\ 1 Laboratorio de Macroalgas, Centro Interdisciplinario de Ciencias Marinas (CICIMAR-IPN). Apdo. Postal 592, La \\ Paz, Baja California Sur, México C. P. 23090. Tel. (52) 61212 25344, Fax: (52) 6121225322. \\ 2 Departamento de Zootecnia, Universidad Autónoma de Baja California Sur. Km 5 Carr. al Sur. La Paz, B. C. S., \\ México C.P. 23050. \\ 3 Departamento de Nutrición Animal. Instituto Nacional de Ciencias Médicas y de la Nutrición Salvador Zubirán. Vasco \\ de Quiroga No. 15, México D. F. 14000. \\ * Becario de COFAA y EDI \\ Direcciones electrónicas: mcasasv@hotmail.com; hehi@uabcs.mx; amarin@ipn.mx; raguilar@ipn.mx; \\ chernan@1ycos.com; isrnacho@hotmail.com; silvicarrillo3@hotmail.com
}

Recibido 25-II-2005. Corregido 22-VIII-2005. Aceptado 05-IX-2005.

\begin{abstract}
The seaweed Sargassum (Sargassaceae) as tropical alternative for goats' feeding. The nutritive value of seaweed (Sargassum spp.) was studied in Baja California Sur, Mexico. Twenty female Nubian goats (43-weeks old) were randomly distributed into two groups of 10 goats each and were housed in individual pens. One group was fed with a control diet and the other with a diet supplemented with $25 \%$ of Sargassum spp. Feed and water intake were recorded daily and individually for 60 days. The weight of each goat was recorded every 15 days. The nutritional content of Sargassum spp. was $89 \%$ dry mater, $8 \%$ crude protein, $31 \%$ ash, $2 \%$ ether extract, and $39 \%$ carbohydrates. Fiber fractions, minerals, vitamins, fatty acids, and antinutritional factors were also determined. There were no significant differences in body weight $(8.6 \mathrm{~kg}$ control and $9 \mathrm{~kg}$ experimental), feed intake $(1.3 \mathrm{~kg}$ control and $1.6 \mathrm{~kg}$ experimental), and feed conversion rate (11.1 control and 12.6 experimental). Water consumption was greater in the goats that ate the Sargassum diet (5.3 1). From these results, Sargassum spp. can be considered as an alternative feedstuff for goats. Rev. Biol. Trop. 54(1): 83-92. Epub 2006 Mar 31.
\end{abstract}

Key words: marine algae, Sargassum spp., goats, animal feeding, chemical composition.

Las algas del género Sargassum C. Agardh forman grandes mantos en aguas tropicales y subtropicales alrededor del mundo, crecen en playas con sustrato rocoso, piedras y cantos rodados (Ganzon-Fortes et al. 1993). En México, se les encuentra en forma muy abundante en todas sus costas; particularmente en la costa oeste del Golfo de California se han estimado 183000 toneladas (peso húmedo) cosechables (Hernández et al. 1990, Casas et al. 1993, Pacheco et al. 1998).

La harina de Sargassum ha sido empleada como suplemento en dietas para gallinas ponedoras mejorando la calidad del huevo y disminuyendo el contenido de colesterol (Meza 1998), recientemente Marín et al. (2003) la utilizaron en la alimentación de ovejas con buenos resultados a nivel metabólico y en los parámetros productivos de estos animales. Algunos estudios indican que estas algas son una buena fuente de minerales, carbohidratos y de algunos aminoácidos esenciales como la arginina, triptofano y fenilalanina; y no se le han detectado factores antinutricios como glucósidos cianogénicos, saponinas y taninos (Carrillo et al. 1992). Los estudios sobre el uso de las algas en la alimentación de cabras son muy escasos, Ventura y Castañón (1998) 
evaluaron el valor nutricional de Ulva lactuca Linnaeus para cabras, determinan una degradación en rumen de $33.5 \%$ de materia orgánica y de $9.6 \%$ de proteína, designando a esta alga como un forraje de calidad media para cabras.

En la región norte de México se presentan sequías cíclicas que ocasionan una disminución significativa en la disponibilidad del forraje de agostadero, por ende las pérdidas en los hatos ganaderos son cuantiosas. Aun así, en esta región la crianza de ganado menor, como el caprino, se lleva a cabo de manera extensiva (aporta el 26\% de la carne de canal y el 68\% de la leche del país) dada la aptitud de estas tierras para agostadero y la adaptación de esta especie animal a climas áridos y semiáridos y a la incorporación de nuevos ingredientes en su alimentación (Genin y Pijoan 1993). Esto ha creado la necesidad de buscar fuentes alternas de forrajes que permitan mantener al ganado caprino durante las duras épocas de sequía y que favorezcan la productividad de este ganado en la región. Las algas marinas del género Sargassum son una alternativa interesante para lograr este fin. En este trabajo se plantearon como objetivos: a) determinar la composición química de Sargassum spp. y b) evaluar el efecto de suplementar la ración con $25 \%$ del alga sobre el comportamiento productivo de cabras en crecimiento.

\section{MATERIAL Y MÉTODOS}

\section{Recolección del alga y análisis químico:} La recolección de Sargassum spp., se realizó en el mes de mayo del 2002 en la playa de San Juan de la Costa, dentro de la Bahía de La Paz en Baja California Sur, México. Área con gran abundancia de este recurso, las especies de Sargassum se encuentran en la siguiente proporción: S. sinicola Setchell et Gardner 1924 (70\%), S. herporizum Setchell et Gardner 1924 (26\%) y S. lapazeanum Setchell et Gardner 1924 (10\%) Cruz et al. (1998). La bahía se localiza entre $\operatorname{los} 24^{\circ} 27^{\prime}$ y $24^{\circ} 06^{\prime} \mathrm{N}$ y $\operatorname{los}$ $110^{\circ} 18^{\prime}$ y $110^{\circ} 40^{\prime} \mathrm{W}$. Las algas se recolectaron manualmente, se esparcieron sobre una plancha de cemento para su secado al sol durante dos días y se molieron en un molino de martillos (Jerza modelo L) para obtener la harina. Por cuarteo se tomo una submuestra de un $1 \mathrm{~kg}$ para realizar los análisis químicos, esta fue molida en un molino de cuchillas (Thomas Scientific modelo 5KH39QN5525) usando una malla de $1 \mathrm{~mm}$. Los análisis se realizaron por triplicado.

Se llevaron a cabo los siguientes análisis químicos: humedad (en una estufa de secado a $60^{\circ} \mathrm{C}$ a peso constante), cenizas (en una mufla eléctrica a $550^{\circ} \mathrm{C}$ ) y extracto etéreo (en un aparato Soxhlet) de acuerdo a los métodos establecidos por la Association of Analytical Chemistry (A.O.A.C. 1999). El contenido de nitrógeno total fue determinado usando el método de micro-Kjeldahl (A.O.A.C. 1999); para calcular el contenido de proteína se utilizó un factor de conversión de 6.25. Las fracciones de fibra fueron analizadas de acuerdo con el método de Van Soest descrito por Tejada (1985). En este método para determinar la fibra neutro detergente (FND) se utiliza el lauril sulfato de sodio para romper la pared celular; este valor corresponde a fibra total. Para determinar la fibra ácido detergente (ADF), la harina fue tratada con bromuro de cetil trimetilamonio para separar la hemicelulosa y el contenido celular. Para el análisis de los minerales la harina fue sometida a una digestión ácida, el $\mathrm{Ca}, \mathrm{Mg}, \mathrm{Na}, \mathrm{K}, \mathrm{Fe}, \mathrm{Mn}, \mathrm{Pb}$ y Cu fueron determinados a través de un espectrofotómetro de absorción atómica y el P por un método colorimétrico (A.O.A.C. 1999).

Los aminoácidos fueron determinados por cromatografía liquida de alta resolución (HPLC), usando un detector fluorescente (Waters modelo 470) y derivatización con carbamato en fase reversa con una columna AccQTaq de Waters. El contenido de aminoácidos fue expresado en $\mathrm{mg} / \mathrm{g}$ de proteína. El procedimiento para cuantificar a las vitaminas A (como retinol), D (como colecalciferol) y E (como tocoferol) fue a través del método descrito por Keller (1988), tiamina por el método No. 97065 del A.O.A.C (1999), riboflavina por el método No. 942.23 del A.O.A.C. (1999) y vitamina C por el método 967.21 del A.O.A.C. (1990). 
Saponinas y glucósidos cianogénicos fueron determinados a través de métodos cualitativos (INNSZ 1984). Taninos mediante una prueba cuantitativa (INNSZ 1984) y alcaloides de acuerdo al método descrito por Domínguez (1979).

Los lípidos totales fueron determinados siguiendo el método descrito por Folch et al. (1957). Este extracto lipídico fue metilado usando trifloruro de boro para cuantificar los ácidos grasos, ácido linoleico (LA C18:2 $\omega 6$ ), $\alpha$-linolenico (ALA C18:3 $\omega 3$ ), araquidónico (AA C20:4 $\omega 6$ ), ecoisapentaenoico (EPA C20:5 (23) y el docosahexaenoico (DHA 22:6 $\omega 3)$, en un cromatógrafo de gases Varian 3400 $\mathrm{CX}$ equipado con un automuestreador y un detector de flama. Se usó una columna DB23 (30 $\mathrm{m}$ x $0.25 \mathrm{~mm}$ de diámetro interno), el gas acarreador empleado fue el nitrógeno con un flujo de $30 \mathrm{ml}$ por minuto, la temperatura de la columna fue de $230^{\circ} \mathrm{C}$, del inyector $150^{\circ} \mathrm{C} \mathrm{y}$ del detector $300^{\circ} \mathrm{C}$. Los tiempos de retención fueron comparados con estándares de cada uno de los ácidos grasos cuantificados.

Formulación y elaboración de dietas: Se formuló y elaboró una dieta testigo con alimentos regionales y una dieta experimental que incluyó 25\% de harina de Sargassum spp., además de los insumos presentes en la dieta testigo (Cuadro 1). La formulación de las raciones y el cálculo del aporte de nutrimentos (Cuadro 2) se hizo a través del programa Nutrion ${ }^{\mathrm{TM}}$ (2002).

Ensayo Experimental: El ensayo experimental se realizó en la Posta Zootécnica de la Universidad Autónoma de Baja California Sur, ubicada en la Cd. de La Paz, B.C.S., México. Esta zona presenta un clima cálido seco. El promedio anual de lluvias en la zona es de $187.6 \mathrm{~mm}$, septiembre es el mes más lluvioso del año $(62.2 \mathrm{~mm})$, la temperatura promedio anual es de $24^{\circ} \mathrm{C}$; presentándose durante los meses de verano temperaturas extremas superiores a $\operatorname{los} 38^{\circ} \mathrm{C}$.

Para el ensayo experimental se utilizaron 20 cabras hembras Nubia, de 43 semanas de edad, con un peso metabólico promedio de $9.5 \mathrm{~kg}$ al inicio del experimento. Las cabras fueron desparasitadas interna y externamente y
CUADRO 1

Composición (\%) de las dietas testigo y experimental

TABLE 1

Composition of the diets used in this study

\begin{tabular}{lcc}
\multicolumn{1}{c}{ Ingrediente } & Testigo & Experimental \\
Maíz blanco & 54.93 & 48.71 \\
Salvado de trigo & 19.54 & 9.38 \\
Rastrojo de maíz & 8.42 & 6.42 \\
Alfalfa & 8.42 & 6.42 \\
Pasta de soya & 6.10 & 3.35 \\
Harina de pescado & 2.58 & ---- \\
Alga Sargassum & ---- & 25.71 \\
Total & 100.00 & 100.00
\end{tabular}

distribuidas aleatoriamente en dos grupos, uno testigo y el otro experimental. Se colocaron en corraletas individuales rústicas, provistas de comederos y bebederos de lámina. Durante 60 días se midió diariamente el consumo voluntario en base seca (diferencia entre el alimento ofrecido y el rechazado) y el consumo de agua, haciendo un resumen de los datos obtenidos de estas variables cada quince días. El peso de cada animal se registró cada 15 días y el peso metabólico se calculó con la siguiente fórmula: $\mathrm{PM}=(\text { Peso de la cabra })^{0.75}$. Para cada una de estas variables se obtuvo un promedio al final del experimento.

Los datos fueron analizados estadísticamente mediante la prueba de t-Student's con una significancia de 0.05 (Steel y Torrie 1985).

\section{RESULTADOS}

La composición química de la harina del alga Sargassum spp. evidencia un alto contenido de material inorgánico y de carbohidratos y un bajo contenido de extracto etéreo. La presencia de ácidos grasos omega 3 en esta alga se considera un aspecto de interés. Respecto 
CUADRO 2

Aporte nutrimental calculado (Programa Nutrion ${ }^{T M}$ 2002) de las dietas testigo y experimental

TABLE 2

Nutrimental value calculated for diet components

\begin{tabular}{|c|c|c|c|c|c|}
\hline Nutrimento & Testigo & Experimental & Nutrimento & Testigo & Experimental \\
\hline EM (Mcal/kg) & 2.62 & 2.62 & Calcio $(\%)$ & 0.035 & 0.14 \\
\hline $\mathrm{ENm}(\mathrm{Mcal} / \mathrm{kg})$ & 1.60 & 1.21 & Fósforo (\%) & 0.050 & 0.38 \\
\hline $\mathrm{ENg}(\mathrm{Mcal} / \mathrm{kg})$ & 1.01 & 0.77 & Metionina(\%) & 0.024 & 0.054 \\
\hline Proteína cruda (\%) & 13.47 & 13.47 & Lisina $(\%)$ & 0.058 & 0.130 \\
\hline Proteína digestible $(\%)$ & 10.35 & 6.05 & Magnesio(\%) & 0.026 & 0.017 \\
\hline Proteína degradable $(\%)$ & 7.43 & 4.57 & Potasio $(\%)$ & 0.180 & 0.071 \\
\hline Proteína sobrepasante $(\%)$ & 45.27 & 35.62 & Sodio $(\%)$ & 0.533 & 0.005 \\
\hline Fibra cruda $(\%)$ & 9.15 & 6.44 & Cloruros (\%) & 0.050 & 0.010 \\
\hline FAD $(\%)$ & 8.16 & 8.90 & Azufre $(\%)$ & 0.010 & 0.018 \\
\hline FND $(\%)$ & 18.38 & 17.32 & Cobalto (mg) & 3.799 & 665.92 \\
\hline Lignina (\%) & 2.19 & 3.27 & Hierro (mg) & 486.94 & 927.53 \\
\hline Hemicelulosa (\%) & 8.60 & 7.25 & Selenio (mg) & 13.35 & 28.43 \\
\hline Materia seca (\%) & 88.08 & 89.81 & & & \\
\hline
\end{tabular}

$\mathrm{EM}=$ Energía metabolizable, $\mathrm{ENm}=$ Energía neta de mantenimiento, $\mathrm{Eng}=$ Energía neta para ganancia de peso, $\mathrm{FAD}=$ Fibra ácido detergente, $\mathrm{FND}=$ Fibra neutro detergente.

a los elementos mayores se aprecia que estas algas son una excelente fuente de potasio, sodio, magnesio, calcio y de elementos traza como el hierro, cobre y zinc. En cuanto al aporte vitamínico resaltan las altas concentraciones de retinol y vitamina $\mathrm{C}$ (Cuadro 3). La composición en aminoácidos de la harina muestra que esta alga es una buena fuente de algunos aminoácidos esenciales como la lisina, fenilalanina, tirosina y treonina; asimismo, presenta altas concentraciones de glutamina y asparagina (Cuadro 4).

Es importante señalar que en estas algas no se detectó la presencia de factores antinutricios como saponinas, alcaloides o glucósidos cianogénicos. En cuanto al ácido tánico se detectaron $1.70 \mathrm{~g} / 100 \mathrm{~g}$ de muestra.
Las tendencias de incremento y decremento en el consumo voluntario de materia seca con relación al peso metabólico de las cabras, resultaron similares entre los grupos a lo largo de los 60 días de experimentación, sin embargo, a los quince y treinta días (periodos 1 y 2) hubo diferencias significativas entre tratamientos ( $\mathrm{p}<0.05)$ (Fig. 1). Respecto a los cambios en el peso vivo metabólico, este fue en ascenso a lo largo del período de estudio sin presentar diferencia significativa entre los tratamientos $(\mathrm{p}>0.05)$ (Fig. 2).

Durante todo el período de experimentación el consumo de agua fue mayor $(p<0.05)$ en las cabras que consumieron dietas complementadas con Sargassum spp. (Fig. 3). 
CUADRO 3

Composición química de la harina del alga Sargassum spp.

TABLE 3

Chemical composition of the seaweed meal Sargassum spp.

Componente químico

Humedad
Cenizas
Proteína ( $\mathrm{N}$ x 6.25)
Extracto etéreo
Fibra cruda
Extracto libre de nitrógeno

Fracciones de fibra

FND

FAD

Celulosa

Hemicelulosa

Lignina

Lípidos totales

Ácidos grasos ${ }^{1}$

Ac. linoleico (18:2 $\omega 6)$

Ác. araquidónico (20:4 $\omega 6)$

Ác. $\alpha$-linolenico (18:3 $\omega 3)$

Ác. eicosapentaenoico (20:5 $\omega 3)$

Ác. docosahexaenoico (22:6 w3)

$$
\begin{gathered}
\mathrm{g} / 100 \mathrm{~g} \\
11.17 \pm 0.08 \\
30.96 \pm 0.27 \\
7.70 \pm 0.23 \\
1.97 \pm 0.01 \\
9.29 \pm 0.43 \\
38.91 \pm 0.50
\end{gathered}
$$

$21.01 \pm 0.90$

$13.18 \pm 1.06$

$7.52 \pm 0.50$

$7.82 \pm 1.65$

$7.00 \pm 0.97$

$9.54 \pm 0.62$

$19.17 \pm 1.51$

$11.63 \pm 0.54$

$4.39 \pm 0.32$

$0.46 \pm 0.12$

1 Los ácidos grasos se expresan en mg/100 g de muestra.

Al final de los 60 días de experimentación los datos promedio obtenidos no mostraron diferencia significativa entre tratamientos en el peso corporal, el consumo de alimento y la conversión alimenticia $(\mathrm{p}>0.05)$ (Cuadro 5).

$\mathrm{mg} / 100 \mathrm{~g}$

Minerales

Sodio

$2066.80 \pm 139.60$

Potasio

$6800.40 \pm 192.10$

Calcio

$500.70 \pm 37.60$

Magnesio

$701.40 \pm 11.40$

Fósforo

$44.90 \pm 3.00$

Manganeso

$5.30 \pm 0.03$

Zinc

$0.98 \pm 0.04$

Hierro

Cobre

$41.20 \pm 3.10$

$0.66 \pm 0.05$

Plomo

$0.20 \pm 0.02$

Vitaminas

\section{Colecalciferol}

6.36

Tocoferol

17.25

Ácido ascórbico

46.25

Tiamina

0.17

Riboflavina

1.18

Retinol (UI)

821.5

\section{DISCUSIÓN}

Las algas marinas del género Sargassum pertenecientes al Orden de las Fucales, por su composición química, son consideradas por 
CUADRO 4

Composición aminoacídica de la harina del alga

Sargassum spp.

TABLE 4

Aminoacids of the seaweed meal Sargassum spp.

Aminoácido

Isoleucina

Leucina

Lisina

Metionina

Cistina

Fenilalanina

Tirosina

2.97

Treonina

2.95

Valina

4.24

Arginina

5.35

Histidina

1.31

Alanina

4.97

Asparagina

6.07

Glutamina

10.23

Glicina

3.28

Prolina

Serina

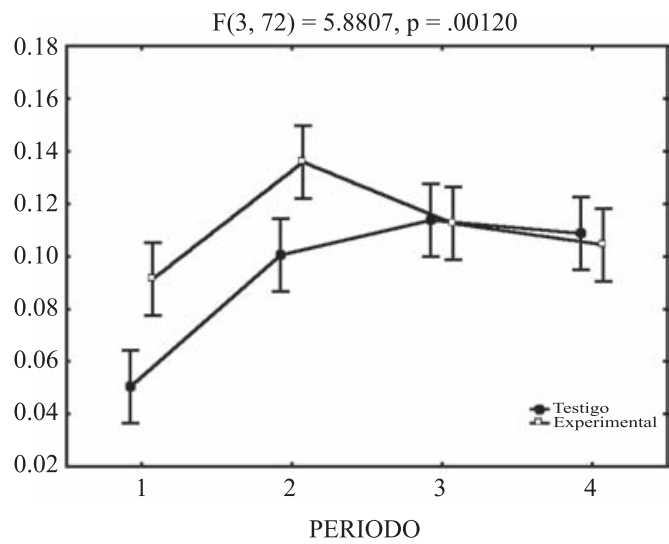

Fig. 1. Consumo voluntario de materia seca en base al peso metabólico de las cabras en los cuatro períodos del experimento.

Fig. 1. Voluntary intake of dry matter on basis metabolic weight during the four experimental periods.

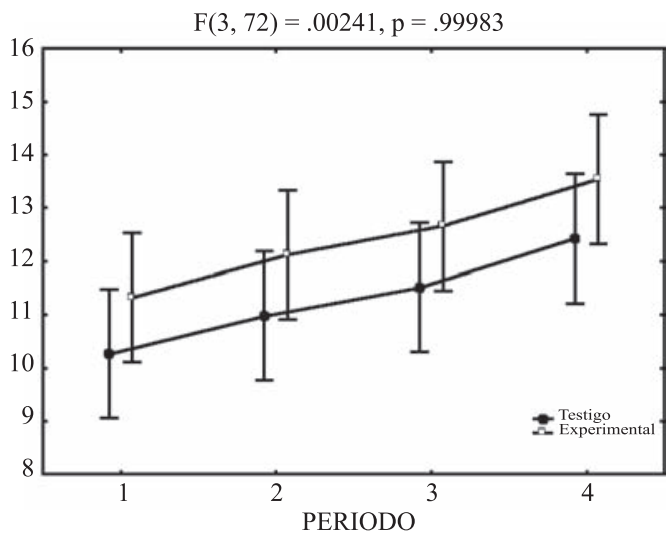

Fig. 2. Peso metabólico de las cabras en los cuatro períodos del experimento.

Fig. 2. Metabolic weight of the goats during the four experimental periods.

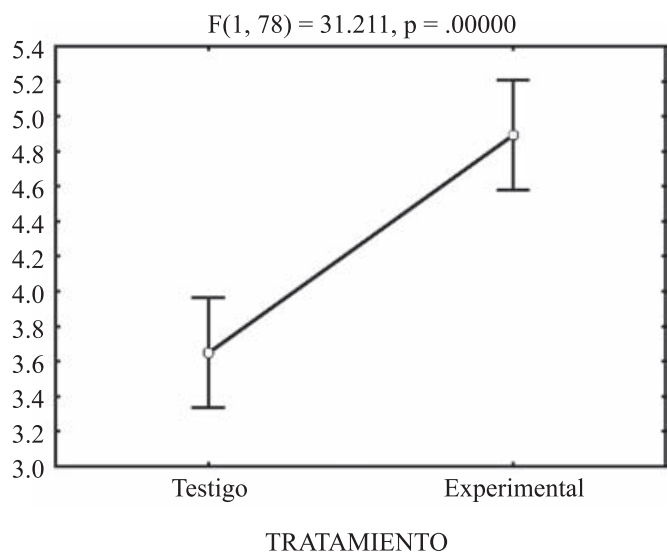

Fig. 3. Consumo de agua de las cabras en el experimento

Fig. 3. Water intake throughout the experiment.

el National Research Council como alimentos energéticos ya que contienen menos de un $20 \%$ de proteína, menos del $18 \%$ de fibra cruda y menos de un $35 \%$ de paredes celulares (o de fibra neutro detergente) (N.R.C. 1981). En este mismo grupo están incluidos forrajes como el arroz, la avena, el centeno, la cebada, el sorgo, el trigo, el maíz y la remolacha azucarera.

El elevado contenido de materia inorgánica se debe a la capacidad que tienen las algas para absorber de un modo selectivo elementos minerales del mar a través de sus polisacáridos 
CUADRO 5

Comportamiento de las cabras durante los 60 dias de experimentación

TABLE 5

Performance of the goats throughout the study

\begin{tabular}{lccc}
\multicolumn{1}{c}{ Variable } & Testigo & Experimental & EEM \\
Peso corporal $(\mathrm{kg})$ & $8.64 \pm 3.38^{\mathrm{a}}$ & $9.08 \pm 3.43^{\mathrm{a}}$ & 1.084 \\
Consumo de alimento $(\mathrm{kg})$ & $1.31 \pm 0.31^{\mathrm{a}}$ & $1.63 \pm 0.38^{\mathrm{a}}$ & 0.120 \\
Conversión alimenticia & $11.11 \pm 7.02^{\mathrm{a}}$ & $12.64 \pm 6.73^{\mathrm{a}}$ & 2.128 \\
Consumo de agua (1) & $3.77 \pm 0.68^{\mathrm{a}}$ & $5.13 \pm 0.98^{\mathrm{b}}$ & 0.309
\end{tabular}

Se presenta media y error estándar de cada tratamiento.

$\mathrm{EEM}=$ Error estándar de la media.

En cada fila literales distintas indican diferencia significativa $\mathrm{p}<0.05$.

superficiales, y constituye un aspecto muy particular de estos organismos marinos (JiménezEscrig y Goñi 1999, Carrillo et al. 2002). Asimismo, es importante señalar que en el extracto libre de nitrógeno se incluyen polisacáridos de reserva y estructurales, comunes en las algas pardas, como los alginatos, furanos y celulosa (Jiménez-Escrig y Goñi 1999). La mayor parte de estos polisacáridos pueden ser considerados como compuestos fibrosos.

El contenido de paredes celulares y de FAD obtenidos en las algas Sargassum spp. es similar a los datos obtenidos por Ventura y Castañón (1998) en el alga verde Ulva lactuca (33\% y $15 \%)$, así como los de la avena $(30 \%$ y $14 \%)$ y el arroz forrajeros (26\% y $13 \%)$ (N.R.C. 2001).

La presencia de los ácidos grasos de las series $\omega 3$ y $\omega 6$ en las algas es de interés. En el caso particular de los $\omega 3$, son muchos los beneficios que estos nutrimentos brindan al humano, por ello se ha buscado la manera de utilizar productos de origen animal (huevo, leche, carne de pollo, de res y de cerdo) como vehículos para hacer llegar al consumidor estos beneficios (Simopoulos 1998). En el caso de la carne y leche de cabras, su composición en ácidos grasos también puede ser modificada a través de la dieta, al igual que sucede en las otras especies animales; y aunque son muy pocos los trabajos que al respecto se han realizado, resulta de interés saber que los ácidos grasos presentes en la harina de Sargassum spp. pueden ser depositados en el músculo del animal (Banskalieva et al. 2000).

$\mathrm{Su}$ alto contenido en elementos mayores y traza, es de gran importancia, debido a que en general las raciones que se suministran al ganado caprino son deficientes en minerales y es necesario suplementarlas. En el caso del hierro las concentraciones obtenidas en la harina de Sargassum spp. están muy por encima de las cantidades aportadas por otros alimentos energéticos (N.R.C. 1981). Además, una ventaja adicional de incluir algas marinas en la dieta para cabras es el hecho de que, los minerales presentes en las mismas son altamente disponibles por encontrarse en forma orgánica, lo que los hace más asimilables, según lo señalan Chapman y Chapman (1980).

El contenido de vitaminas presente en estas algas resulta interesante, si se toma en cuenta que en general los forrajes son deficientes en estas. De hecho, no poseen vitamina A per se, aun cuando sus precursores como el beta-caroteno si lo está ( $1 \mathrm{mg}$ de beta caroteno en la dieta $=400$ UI vitamina A). La presencia de vitamina $\mathrm{E}$ en la dieta es importante a fin de prevenir en las cabras la enfermedad del músculo blanco; además, al ser transferida esta vitamina a la leche, contribuye a prolongar el tiempo de almacenamiento de la misma debido 
a sus propiedades antioxidantes (N.R.C. 1981). Respecto a las vitaminas del complejo B, aunque estas no son consideradas esenciales en los animales adultos, debido a que estas son sintetizadas por los microorganismos del rumen, en los animales muy jóvenes y en aquellos con cambios muy radicales de alimentación, si es importante proporcionárselas en la dieta (AFRC 1998).

En cuanto a los aminoácidos de esta alga, su contenido de isoleucina, leucina, lisina, metionina, treonina, valina y arginina es similar al del sorgo y a la cebada, por lo que esta harina puede ser un ingrediente útil al formular dietas balanceadas con base en los aminoácidos esenciales para rumiantes (N.R.C. 2001).

Por otra parte, la cantidad de ácido tánico encontrada en las algas fue bajo por lo que no es suficiente para ocasionar efectos negativos en la salud o comportamiento del animal y por el contrario pudiera contribuir a modular la fermentación ruminal (Makkar 2003).

Los datos obtenidos de consumo voluntario en las cabras, indican que las características físicas con que se ofreció el alga marina, no repercutieron sobre esta variable; y que aunque la energía para mantenimiento y ganancia de peso aportadas por la dieta experimental fue menor a la dieta testigo, esto no influyó negativamente sobre el consumo de materia seca y el crecimiento de los animales.

Por el contrario, el incremento en el consumo de la dieta que contenía el alga, fue algo notable aunque no significativa ( $\mathrm{p}>0.05)$ y coincide con lo observado por Al-Shorepy et al. (2001) en corderos suplementados con 1\% de algas marinas. Esto puede atribuirse al hecho de que el alga estimula el consumo de alimento (Tierney y Atema 1988), manteniendo las ganancias de peso similares a las de dietas convencionales.

El consumo de agua se incrementó en el lote experimental, debido al alto contenido de sales en la dieta por efecto de la inclusión de las algas. Los rumiantes son capaces de tolerar un alto consumo de sales en la dieta, incrementando el consumo de agua para regular el balance osmótico en el tracto gastrointestinal (Underwood y Suttle 1999).
El no encontrar diferencia significativa en la ganancia de peso y en el peso metabólico entre el grupo experimental y el testigo, indica que no existen respuestas negativas al incluir $25 \%$ del alga Sargassum spp. en la dieta.

El alga marina Sargassum spp., es un recurso natural muy abundante, que esta disponible para su aprovechamiento sustentable en la alimentación de ganado caprino, debiendo recibir como tratamiento únicamente un secado al sol. Su buen valor nutrimental hace de este recurso una alternativa para alimentar a este ganado principalmente durante las épocas de sequía. Este resultado se puede aplicar en aquellos países donde Sargassum spp. es muy abundante y presentan poca disponibilidad de forrajes de agostadero.

\section{AGRADECIMIENTOS}

Agradecemos a la Fundación PRODUCE Baja California Sur, la cual aportó parte de los fondos; a la Unión Ganadera de Baja California Sur por su apoyo y a Augusto Duarte, quien facilitó los animales para realizar el experimento.

\section{RESUMEN}

Se estudió el valor nutricional de varias algas del género Sargassum. Veinte cabras hembras (Nubia) en crecimiento (43 semanas) se distribuyeron aleatoriamente en dos grupos de 10 cabras y se alojaron en corraletas individuales. Un grupo fue alimentado con una dieta testigo elaborada con insumos regionales y el otro con una dieta que incluyó el 25\% de Sargassum spp. Durante 60 días se midieron diariamente y en forma individual, el alimento y agua consumidos, el peso corporal se cuantificó cada 15 días. La composición química proximal de Sargassum fue de: $89 \%$ materia seca, $7.7 \%$ proteína, $31 \%$ cenizas, $2 \%$ extracto etéreo y $39 \%$ de carbohidratos. También se determinaron las fracciones de fibra, minerales, vitaminas, ácidos grasos y factores antinutricios. No se encontraron diferencias significativas en el peso corporal (8.6 kg grupo control y $9 \mathrm{~kg}$ experimental), alimento consumido $(1.31 \mathrm{~kg}$ grupo control y 1.6 experimental) y tasa de conversión alimenticia (11.1 grupo control y 12.6 experimental) entre los tratamientos. El consumo de agua fue mayor en el grupo alimentado con la dieta que incluyó Sargassum spp (5.1 1). 
De los resultados se deduce que las algas marinas de este género pueden ser utilizadas como un forraje alternativo de mediana calidad para el ganado caprino.

Palabras clave: alga marina, Sargassum spp., cabras, alimento para ganado, composición química.

\section{REFERENCIAS}

AFRC. 1998. The Nutrition of Goat. AFRC Technical Committee on Responses to Nutrients, Report No. 10, $\mathrm{CAB}$ International, Grand London, Londres, Reino Unido $118 \mathrm{p}$.

Al-Shorepy, S.A., G.A. Alhadrami \& I.A. Jamali. 2001. Effect of feeding diet containing seaweed on weight gain and carcass characteristics of indigenous lambs in the United Arab Emirates. Small Rum. Res. 41: 283-287.

A.O.A.C. 1990. Official Methods of Analysis. Association of Analytical Chemists. Washington, D.C. EE.UU. $1141 \mathrm{p}$.

A.O.A.C. 1999. Official Methods of Analysis. Association of Analytical Chemists. 16 th Ed., 5 th revision. Washington, D. C. EEUU 1545 p.

Banskalieva, V., T. Sahlu \& A.L. Goetsch. 2000. Fatty acid composition of goat muscles and fat depots: a review. Small Rum. Res. 37: 255-268.

Carrillo, S., M.I. Castro, F. Pérez-Gil, E. Rosales \& R.E. Manzano. 1992. The seaweed (Sargassum sinicola Setchell \& Gardner) as an alternative for animal feeding. Cuban J. Agric. Sci. 26: 177-181.

Carrillo, S., M. Casas, F. Ramos, F. Pérez-Gil \& I. Sánchez. 2002. Algas marinas de Baja California Sur, México: Valor nutrimental. Arch. Latinoamer. Nutr. 52(4): 115-125.

Casas, M., I. Sánchez \& G. Hernández. 1993. Evaluación de Sargassum spp. en la costa oeste de Bahía Concepción, B. C. S., México. Inv. Mar. CICIMAR 8(2): 61-68.

Chapman, V.J. \& D.J. Chapman. 1980. Seaweeds and their uses. Chapman and Hall, Londres, Reino Unido 334 p.

Cruz, M., M. Casas \& S. Ortega. 1998. Temporal and Spatial Variation of Frondose Benthic Seaweeds in La Paz Bay, B.C.S., Mexico. Bot. Mar. 41: 191-198.

Domínguez, X. 1979. Método de investigación fitoquímica. Limusa. México, D. F., México 281 p.
Folch, J., M. Less \& G. Stanley. 1957. A simple method for the isolation and purification of total lipids from animal tissues. J. Biol. Chem. 226: 497-509.

Ganzon-Fortes, E.T., R.R. Campos \& J. Udarbe. 1993. The use of Philippine seaweeds in agriculture. Appl. Phycol. Forum 10: 6-7.

Genin, D. \& A.P. Pijoan. 1993. Seasonality of goat diet and plant acceptabilities in the coastal scrub of Baja California, México. Small Rum. Res. 10(1): 1-11.

Hernández, G., M. Casas, C. Fajardo, I. Sánchez \& E. Rodríguez. 1990. Evaluación de Sargassum spp. en la Bahía de la Paz, B. C. S., México. Inv. Mar. CICIMAR 5(1): 11-18.

INNSZ. 1984. Manual de técnicas de laboratorio para el análisis de alimentos. Depto. de Ciencia y Tecnología de los alimentos. Instituto Nacional de la Nutrición Salvador Zubirán. México D. F., México 175 p.

Jiménez-Escrig, A. \& I. Goñi. 1999. Evaluación nutricional y efectos fisiológicos de macroalgas marinas comestibles. Arch. Latinoamer. Nutr. (49)2: 114-120.

Keller, H.E. 1988. Analytical Methods for Vitamins and Carotenoids in Feed. Roche Vitamins and Fine Chemicals Division, Roche Edition 2101. F. Hoffmann-La Roche, Basilea, Suiza. 99 p.

Makkar, H.P.S. 2003. Effects and fatty of tannins in ruminants animals, adaptation to tannins, and strategies to overcome detrimental effects of feeding tannin-rich feeds. Small Rum. Res. 49: 241-256.

Marín, A., M. Casas, S. Carrillo, H. Hernández \& A. Monroy. 2003. Performance of sheep fed rations with Sargassum spp. sea algae. Cuban J. Agric. Sci. 37(2): 119-123.

Meza, A.M.I. 1998. Impacto sobre la calidad del huevo al incluir algas marinas en raciones para gallinas ponedoras. Tesis de Maestría. Facultad de Medicina Veterinaria y Zootecnia. Universidad Nacional Autónoma de México, México D.F., México 97 p.

N.R.C. 1981. Nutrient requirements of goats National Academy, Washington, D.C., EE.UU. 91 p.

N.R.C. 2001. Nutrient requirements of dairy cattle. National Academy, Washington, D.C., EE.UU. 202 p.

Nutrion. 2002. Manual de Operación Nutrion 5 Básico. Version 2002. Comercializadora de Software S. A. de C. V., México, D.F., México 25 p.

Pacheco, R.I., J. Zertuche, B. Chee \& R. Blanco. 1998. Distribution and quantification of Sargassum beds along the west coast of the Gulf of California, México. Bot. Mar. 41: 203-208. 
Simopoulos, A.P. 1998. The Return of $\omega 3$ Fatty Acids into the Food Supply. I. Land-based Animal Food Products and Their Health Effects. Karger Ed., Basel. Switzerland. $240 \mathrm{p}$.

Steel, R.G. \& J.H. Torrie. 1988. Bioestadística: Principios y procedimientos. McGraw-Hill. México, D.F., México $622 \mathrm{p}$.

Tejada, H.I. 1985. Manual de laboratorio para análisis de ingredientes en la alimentación animal. Patronato de Apoyo a la Investigación y Experimentación Pecuaria en México. Instituto Nacional de Investigaciones Forestales y Agropecuarias (INIFAP), Secretaría de
Agricultura y Recursos Hidráulicos (SARH), México, D.F., México. 714 p.

Tierney, A.J. \& J. Atema. 1988. Behavioral responses of cryfish (Orconectes virilis and Orconectes rusticus) to chemical feeding stimulants. J. Chem. Ecol. 14(1): 123-132.

Underwood, E.J. \& N.F. Suttle. 1999. The Mineral Nutrition of Livestock. CAB International, Wallinford, Oxon, Reino Unido 477-512.

Ventura, M.R. \& I. Castañón. 1998. The nutritive value of seaweed (Ulva lactuca) for goats. Small Rum. Res. 29: $325-327$. 